\title{
Hidden Scars in Depression? Implicit and Explicit Self-Associations Following Recurrent Depressive Episodes
}

\author{
Hermien J. Elgersma, Klaske A. Glashouwer, \\ and Claudi L. H. Bockting \\ University of Groningen
}

\author{
Brenda W. J. H. Penninx \\ VU University Medical Centre and Leiden University \\ Medical Centre
}

Peter J. de Jong

University of Groningen

\begin{abstract}
To help explain the recurrent nature of major depressive disorder, we tested the hypothesis that depressive episodes and/or the duration of depressive symptoms may give rise to persistent dysfunctional implicit and/or more explicit self-associations, which in turn may place people at risk for the recurrence of symptoms. We therefore examined, in the context of the Netherlands Study of Depression and Anxiety, whether the strength of self-depressed associations at baseline was related to the number of past episodes (retrospective analysis; $n=666$ ), and whether the duration of symptoms between baseline and follow-up predicted self-depressed associations at 2-year follow-up (prospective analysis; $n=726$ ). The lifetime Composite International Diagnostic Interviews and Life Chart Interview were used to index the number of depressive episodes; the Implicit Association Test and its explicit equivalent were used to index self-associations. Consistent with the hypothesis that self-depressed associations strengthen following prolonged activation of negative self-associations during depressive episodes, individuals' implicit and explicit self-depressed associations correlated positively both with the number of prior depressive episodes at baseline and with the duration of depressive symptoms between baseline and 2-year follow-up. There was evidence that these relationships held, particularly in the prospective study, even when controlling for neuroticism and current depressive symptoms, whereas the retrospective relationship between number of episodes and implicit self-associations fell just short of significance.
\end{abstract}

Keywords: longitudinal study, implicit self-depressed associations, explicit self-depressed associations, depression, recurrence

Major depressive disorder (MDD) causes suffering in the individual and his or her environment, and contributes to high societal and health care costs (Ormel et al., 2008; Smit et al., 2006). In 2030, MDD is expected to be at the top of the list for the World Health Organization in terms of burden of disease. Notably, its recurrent nature contributes to the disability and health care costs of MDD (Mathers \& Loncar, 2006). The chance of recurrence reaches $90 \%$ in patients with three or more episodes (Mueller et al., 1999; Judd et al., 1998). Consequently, it is very important to obtain a better understanding of the processes that increase vulnerability to relapse and recurrence in depression.
Cognitive models emphasize the relevance of dysfunctional attitudes toward the self in the onset and recurrence of depressive episodes (Clark, Beck, \& Alford, 1999). Dual-process models point to the importance of distinguishing between more explicit and more automatic (implicit) attitudes in this respect (e.g., Gawronski \& Bodenhausen, 2006; Haeffel et al., 2007). To help explain the onset, course, and recurrence of depression, Beevers (2005) applied this dual-process perspective to the cognitive vulnerability to depression. He proposed that, in response to a stressful life event, two sets of processes determine how that event will be evaluated. First, by default, the implicit processing system is
Hermien J. Elgersma, Klaske A. Glashouwer, and Claudi L. H. Bockting, Department of Clinical Psychology, University of Groningen, Groningen, The Netherlands; Brenda W. J. H. Penninx, Department of Psychiatry/EMGO Institute, VU University Medical Centre, Amsterdam, The Netherlands, Department of Psychiatry, Leiden University Medical Centre, Leiden, The Netherlands; and Peter J. de Jong, Department of Clinical Psychology, University of Groningen, Groningen, The Netherlands.

Supported by ZonMw (OOG) (Grant 100000-2035) and Accare Drenthe/Overijssel. Netherlands Study of Depression and Anxiety infrastructure supported by the Netherlands Organisation for Health Research and Development (Grant 10-000-1002) and by participating universities and men- tal health care organizations (VU University Medical Center, GGZ inGeest, Arkin, Leiden University Medical Center, GGZ Rivierduinen, University Medical Center Groningen, University of Groningen, Lentis, GGZ Friesland, GGZ Drenthe, Scientific Institute for Quality of Health Care [IQ Health Care]), Netherlands Institute for Health Services Research [NIVEL], and Netherlands Institute of Mental Health and Addiction [Trimbos]). We thank Bert Hoekzema for technical support, and Maggie Stroebe for her helpful comments and suggestions.

Correspondence concerning this article should be addressed to Hermien J. Elgersma, Department of Clinical Psychology, University of Groningen, Grote Kruisstraat 2/1, 9712 TS Groningen, The Netherlands. E-mail: h.j.elgersma@rug.nl 
activated, which consists of associative memory networks. Implicit associations are seen as the direct activation of simple associations in this memory network, which occur unintentionally and fast. In individuals vulnerable to depression, implicit associations are assumed to be negative and to immediately lead to negative cognitive and affective responses. Second, the reflective processing system can be triggered, which may or may not lead to an adjustment of the initial negative response of the implicit system. The crucial difference between these different kinds of mental processes is that the reflective system is generally concerned with the validation of cognitions and requires time and resources. In contrast, the activation of associations occurs nonintentionally and whether or not a person considers these associations to be true or false. Over time, a negative feedback loop can develop between associative processing and symptoms of depression when people fail to correct their dysfunctional implicit associations (e.g., because of a lack of available resources, and/or dysfunctional reflective strategies). For instance, when a person makes a mistake at his or her job, this may directly elicit an association between "me" and "hopeless." Given sufficient time and resources, such an association can be corrected into something like "Everyone makes mistakes. I feel really upset by it, but I can learn from my mistake and will do it differently next time." However, when this person has insufficient cognitive resources available, it becomes increasingly difficult to disengage from negative thinking, eventually resulting in a depressive episode.

Building further on this model, it was proposed that the repeated activation of negative associations during a depressive episode might result in an associative memory network in which the self becomes increasingly linked to negative attributes (Risch et al., 2010). Furthermore, by repeated activation of negative implicit self-associations during several depressive episodes, or by longer exposure to depressive symptoms, dysfunctional self-associations might become increasingly ingrained. By ingraining dysfunctional self-associations, a feedback loop might be formed between associative processing and depressive symptoms: dysfunctional selfassociations can become more easily (automatically) activated, even by mild stress or mild negative mood states, and may turn into a "hidden scar": a chronic vulnerability factor that may help explain the recurrent nature of depressive disorder.

In line with this view, previous cross-sectional research has shown that patients remitted from MDD have substantially stronger explicit as well as implicit self-depressed associations than never depressed individuals (Glashouwer \& de Jong, 2010). Yet, in apparent contrast with this, a relatively small scale study contrasting currently depressed patients, remitted patients, and never depressed participants failed to find a similar difference in (implicit) self-esteem between remitted and never depressed participants (Risch et al., 2010). In an attempt to explain the unexpected absence of a significant difference between never depressed participants and remitted patients, it was argued that in this study remitted patients with relatively few prior episodes might have been combined with remitted patients with relatively many prior episodes. If negative self-associations become increasingly ingrained following successive depressive episodes, implicit negative self-associations might only be evident in participants with relatively many previous episodes. Interestingly and consistent with this post hoc explanation, additional analyses showed that individuals with relatively many episodes displayed lower (im- plicit) self-esteem than individuals with relatively few episodes (Risch et al., 2010).

To establish whether these promising exploratory results reflect a reliable and theoretically relevant finding, it would be important to put them to a more stringent test in an independent sample. Therefore, the first aim of the present study was to establish whether a relationship between the number of previous episodes and the strength of negative self-associations could be found in the Netherlands Study of Depression and Anxiety (NESDA; Penninx et al., 2008). The latter study is suited to this purpose, because it is a large scale, longitudinal cohort study, designed to examine the long-term course and consequences of depressive (and anxiety) disorders. In addition, it is important to establish whether this relationship_-if confirmed-could be explained by well-known psychopathological risk factors. Therefore, we also examined to what extent the relationship between the number of previous episodes and current strength of self-depressed associations (if present) is independent of neuroticism (Spinhoven et al., 2011a, 2011b).

Examination of the time course of relationships between depressive episodes and self-depressed associations has not been given much attention in previous investigations. Yet, in our view, this is important to study: even if the current study were to find a robust relationship between the number of prior episodes and the strength of individuals' negative self-associations, it cannot be ruled out that the relatively negative self-associations in fact represent a premorbid characteristic of people who suffered from relatively many depressive episodes rather than a consequence of repeated depressive episodes. In the present study, we therefore complemented the retrospective approach of Risch et al. (2010) with a prospective design that allowed us to control for the strength of baseline self-associations. More specifically, we examined whether the duration of symptoms between baseline and the 2-year follow-up had predictive value for the self-depressed associations at follow-up over and above the initial self-depressed associations during the baseline assessment.

To explore whether the relationship between the number of prior episodes/duration of symptoms and self-depressed associations would be restricted to the self-report level or would also be evident at the implicit level, we assessed both implicit and explicit selfdepressed associations. This approach also allowed us to test the extent to which the relationship between the number of prior episodes/duration of symptoms and implicit self-associations is independent of the relationship between the number of episodes/ duration of symptoms and explicit self-associations (and vice versa).

In short, based on the preliminary findings of Risch et al. (2010), we expected that: (a) a higher number of prior depressive episodes would be associated with stronger (explicit and implicit) selfdepressed associations; and (b) longer duration of depressive symptoms between the baseline assessment and follow-up would be associated with stronger (explicit and implicit) self-depressed associations. Strong explicit self-depressed associations are assumed to become increasingly ingrained and, therefore, also evident at the more implicit level, whereas the presence of relatively strong implicit self-depressed associations will, in turn, lower the threshold for activating explicit self-depressed associations. Given this alleged reciprocal relationship between explicit and implicit self-depressed associations, we anticipated that the relationship 
between the number of previous episodes/duration of symptoms and self-associations would be largely similar for implicit and explicit self-depressed associations.

\section{Method}

This study was part of NESDA (Penninx et al., 2008), which is an ongoing multicenter, longitudinal cohort study, designed to examine the long-term course and consequences of anxiety and depressive disorders (see also http://www.nesda.nl). The NESDA study protocol was approved centrally by the Ethical Review Board of VU Medical Centre Amsterdam and by the local review boards of each participating center. All participants provided written informed consent.

\section{Participants}

Participants of the NESDA study were recruited from the general population, in general practices and in mental health care institutions. Uniform inclusion and exclusion criteria were used across all recruitment settings. A general inclusion criterion was an age of 18-65 years. The two exclusion criteria were: (a) primary clinical diagnosis of a psychiatric disorder not the subject of NESDA that would largely affect course trajectory: psychotic disorder, obsessive-compulsive disorder, bipolar disorder, or severe addiction disorder; and (b) not being fluent in Dutch. For a more detailed study description, see Penninx et al. (2008). The present study concerned baseline and 2-year follow-up measurements, conducted from September 2004 until April 2009. In total 2,981 participants were included, of whom 652 were nonclinical controls without present or past depressive and/or anxiety diagnosis.

For the retrospective assessment, we selected participants who had one or more depressive diagnoses (e.g., MDD, dysthymia) in the past, but were remitted at baseline $(n=815)$. We restricted this analysis to remitted participants to prevent the influence of current depression on participants' implicit and explicit self-associations (e.g., Glashouwer \& de Jong, 2010). Of these 815 participants, 752 completed the Implicit Association Test (IAT) for depression. There were no significant differences in demographic variables, number of prior depressive episodes, depression IAT, or explicit self-associations between the 752 participants who completed a depression IAT and the 63 participants who did not. In the retrospective approach, the data reflecting the number of prior episodes were missing for 79 participants, and, for another seven participants, the scores on the Inventory of Depressive Symptoms SelfReport (IDS-SR) were missing. Therefore, the final sample included in the retrospective analyses consisted of 666 participants.

After 2 years, a face-to-face follow-up assessment was conducted with a response of $87.1 \%(n=2,596)$. Nonresponse was significantly higher among those of younger age, lower education, non-European ancestry, and depressive disorder, but was not associated with gender (Lamers et al., 2012). The prospective analyses testing the relationship between the duration of depressive symptoms between baseline and 2-year follow-up and selfassociations were restricted to participants who met the criteria for MDD at baseline and/or at follow-up because information on the duration of depressive symptoms was available only for these participants in NESDA $(n=979)$. Of these, 762 participants completed both IAT assessments. There were no significant differences in demographic variables, baseline implicit or explicit measures, or duration of symptoms between the 762 participants who completed both depression IATs and the 217 participants who did not. In the prospective approach, information about the duration of symptoms was missing for 21 participants, data for the IDS were missing for another 11 participants, and data for the NEOFive Factor Inventory (NEO-FFI) for four participants (final $n=$ 726). Note that participants who were remitted at baseline and were depressed at follow-up were part of both samples; accordingly, 87 participants were included in both analyses.

\section{Measurements}

Diagnostic assessment. Depressive disorders were determined using the lifetime Composite International Diagnostic Interviews (CIDI; World Health Organization, Version 2.1; Robins et al., 1988; Wacker, Battegay, Muellejans, \& Schlosser, 2006). The CIDI classifies diagnoses according to criteria of the Diagnostic and Statistical Manual of Mental Disorders, Fourth Edition (DSM-IV; American Psychiatric Association, 2001). People were considered remitted when they no longer met the criteria for MDD, but had experienced one or more depressive episodes in the past.

The number of prior depressive episodes at baseline, the duration of depressive symptoms between baseline and follow-up, and recurrence (having a depressive episode during the 2-year period) were determined using two sources of data collected during both assessments: (a) CIDI interview and (b) Life Chart Interview (LCI). The CIDI interview determined presence of DSM-IV classified depressive disorders at baseline assessment or 2-year followup. The LCI was completed for all persons with detected depressive symptoms in the CIDI interview. Life events were recalled to refresh memory using a calendar method, after which the presence of depressive symptoms at each month during this 2-year period was determined (Lyketsos, Nestadt, Cwi, \& Heithoff, 1994). In addition, severity was assessed (ranging from no or minimal severity to mild, moderate, severe or very severe) for each month in which symptoms were reported. Symptoms on the LCI were considered to be present when at least of mild severity (Penninx et al., 2011). The number of prior depressive episodes at baseline was determined on the basis of the results of the CIDI interview and the LCI at the baseline assessment. In the assessment at follow-up, participants were asked (as part of the LCI) whether they had experienced depressive symptoms during each month. If so, they were asked for the burden of symptoms in that particular month. To establish duration of depressive symptoms between baseline and follow-up assessments, the total number of months with depressive symptoms was counted and divided by the number of months of the follow-up period.

Questionnaires. Neuroticism was measured at baseline using the Neuroticism domain of the NEO-FFI (McCrae \& Costa, 2004). We used total scores on the Neuroticism domain as our index. Severity of depressive symptoms was assessed using the 30-item IDS-SR (Rush, Gullion, Basco, Jarrett, \& Trivedi, 1996). We used total scores of the IDS as an index for the severity of depression.

Implicit Association Test. The IAT is a computerized reaction time (RT) task originally designed by Greenwald, McGhee, and Schwartz (1998) to measure the relative strengths of implicit associations between two contrasted target concepts and two attri- 
bute concepts. Words from all four concept categories appear in mixed order in the middle of a computer screen, and participants are instructed to sort them with a left or a right response key. The premise is that sorting becomes easier when a target and an attribute that share the same response key are strongly associated than when they are weakly associated. The category labels are visible in the upper-left and right-hand corners of the screen during the whole task (for an example, visit https://implicit.harvard.edu/ implicit). The target labels were "me" and "other." The attribute labels were "depressed" and "elated." Each category consisted of five stimuli (see Appendix A). The IAT consists of two critical test blocks, preceded by practice blocks (see Table 1). In one test block, "me" and "depressed" (and "other" and "elated") share the same response key, whereas in the other test block "me" and "elated" (and "other" and "depressed") shared the response key. Before the start of a new sorting task, written instructions were presented on screen. After a correct response, the next stimulus was presented after $500 \mathrm{~ms}$. After an incorrect response, the Dutch word FOUT! (wrong) appeared shortly above the stimulus. Meanwhile, the stimulus remained on the screen until the correct response was given. The order of the category combinations was fixed across participants to reduce method variance. The split-half reliability of the present IAT was good, with Spearman-Brown corrected correlations between test-halves of .87 .

Explicit self-associations. To obtain explicit self-associations equivalent to the implicit self-associations, participants rated all IAT attribute stimuli on a 5-point scale $(1=$ hardly/not at all and 5 = very much). The instruction was: "For each word please indicate to what extent you think it generally applies to you" (cf. Back, Schmukle, \& Egloff, 2009). Mean ratings of depressed IAT stimuli were subtracted from mean ratings of elated IAT stimuli. Positive effects indicate relatively strong explicit associations between "me" and "elated." Internal consistencies of explicit selfassociation measures were excellent ( $\alpha=.95$ for the difference scores of depressed and elated words).

\section{Procedure}

The assessments at baseline and follow-up were similar; they lasted between 3 and 5 hours, and were conducted on a single day. During the assessments, participants first completed the CIDI, then carried out the IAT, and finally completed the explicit ratings. Other measurements were collected in between and afterward, but they were not of interest for the present study (e.g., before the depression IAT, participants filled in the anxiety IAT; see Penninx et al., 2008, for a detailed description). Respondents were compensated with a €15 gift certificate, and travel expenses were reimbursed.

Table 1

Arrangement of Implicit Association Test Blocks

\begin{tabular}{lllc}
\hline \multicolumn{1}{c}{ Block } & Left label(s) & Right label(s) & No. of trials \\
\hline 1. Practice & Depressed & Elated & 20 \\
2. Practice & Me/depressed & Other/elated & 20 \\
3. Test & Me/depressed & Other/elated & 60 \\
4. Practice & Elated & Depressed & 20 \\
5. Practice & Me/elated & Other/depressed & 20 \\
6. Test & Me/elated & Other/depressed & 60 \\
\hline
\end{tabular}

Data reduction IAT. IAT scores were computed according to the D measure proposed by Greenwald, Nosek, and Banaji (2003) on the basis of Internet studies. Recent research has shown that the D measure also performed best in a laboratory setting, when evaluated on the basis of a series of psychometric criteria (Glashouwer, Smulders, de Jong, Roefs, \& Wiers, 2013). We report the D4 measure. Following the guidelines, all RTs above $10,000 \mathrm{~ms}$ were discarded, and error trials were replaced with the mean RTs of the correct responses in the block in which the error occurred, plus a penalty of $600 \mathrm{~ms}$. The IAT effect was calculated by subtracting the mean RTs of Block 2 from Block 5 (practice) and Block 3 from Block 6 (test). The means of these two effects were divided by their inclusive standard deviation based on all responses in Blocks 2, 3, 5, and 6. Negative IAT effects indicate relatively fast responses when "me" shared the response key with "depressed."

Statistical analyses. We used single and multiple regression analyses. For the retrospective approach, we assessed the number of prior depressive episodes during the baseline measurement, and used the outcome as a predictor variable. In the longitudinal approach, we used the percentage of time with depressive symptoms between baseline and follow-up as a predictor variable. Participants with a duration greater than 24 months were counted at 24 months (i.e., 100\%). For both the retrospective and prospective analyses, dependent variables in the model were the implicit self-depressed associations (as indexed by the depression IAT) and explicit self-depressed associations (as indexed by the selfreported associations). In the prospective analyses, we also included the baseline depression IAT index as a covariate, to control for the strength of initial self-depressed associations. In both the retrospective and the prospective analyses, we added neuroticism (as indexed by the NEO-FFI Neuroticism domain total score) and depressive symptoms (as indexed by the IDS-SR total score) into the regression models, correcting for "trait" and "state," respectively. To examine whether the relationship between the number of episodes (or the duration of symptoms) and implicit selfassociations was independent of the relationship between the number of episodes (or the duration of symptoms) and explicit selfassociations (and vice versa), we included the implicit and explicit measure into the regression models. Finally, we also included the sociodemographic variables (i.e., age, gender, and years of education) in the analyses as background variables.

\section{Results}

\section{Descriptives}

Demographics, IAT performance, and self-report measures at baseline and follow-up are reported in Table 2. Correlations between the outcome measures at baseline and follow-up are shown in Table 3.

\section{Does the Number of Prior Depressive Episodes Predict Stronger Implicit Self-Depressed Associations at Baseline?}

The number of prior depressive episodes was entered in a single regression model to predict the depression IAT (see Table 4). This was shown to be significant, indicating that more prior depressive 
Table 2

Means (Standard Deviations) for Retrospective and Prospective Groups

\begin{tabular}{lccc}
\hline & & \multicolumn{2}{c}{$\begin{array}{c}\text { Depressed at baseline and/or } \\
\text { depressed at 2-year follow-up } \\
(n=726)\end{array}$} \\
\cline { 3 - 4 } \multicolumn{1}{c}{ Groups } & $\begin{array}{c}\text { Remitted baseline } \\
(n=666)\end{array}$ & Baseline & 2-year follow-up \\
\hline Gender (\% female) & 71.8 & 65.6 & - \\
Age & $42.92(12.90)$ & $42.25(12.51)$ & - \\
Educational level (years) & $12.39(3.16)$ & $11.68(3.21)$ & - \\
No. of episodes & $3.66(8.05)$ & - & $0.16(0.41)$ \\
Depression IAT, D measure & $0.26(0.39)$ & $0.10(0.42)$ & $0.79(1.58)$ \\
Explicit self-depressed associations $^{\mathrm{a}}$ & $1.70(1.29)$ & $0.29(1.62)$ & $24.00(12.17)$ \\
IDS-SR total score $^{\text {Duration of symptoms }}{ }^{\mathrm{b}}$ & $18.12(10.04)$ & - & $43.55(34.67)$ \\
Neuroticism & $36.06(8.04)$ & $41.61(6.91)$ & \\
\hline
\end{tabular}

Note. $\quad$ IAT $=$ Implicit Association Test; IDS $=$ Inventory of Depressive Symptoms-Self-Report; Neuroticism $=$ total score measured at baseline on the Neuroticism domain of the NEO-Five Factor Inventory.

a Positive effects indicate a relatively stronger an automatic or explicit association between "me" and "elated." Note that the D measure can take negative and positive values. ${ }^{\mathrm{b}}$ Percentage of time with symptoms between baseline and 2-year follow-up.

episodes relate to stronger implicit self-depressed associations. ${ }^{1}$ When neuroticism was entered into the model, the relationship between the number of depressive episodes and implicit selfdepressed associations no longer reached the conventional level $(p=.06)$ of significance. This pattern remained virtually unaffected when the IDS-SR was also included in the model to correct for residual depressive symptoms at baseline, and when the explicit self-associations test was included to control for the strength of concurrent explicit self-depressed associations.

\section{Is the Number of Prior Depressive Episodes Predictive for Explicit Self-Depressed Associations at Baseline?}

The number of prior depressive episodes was entered in a single regression model to predict the strength of the explicit selfdepressed associations. This was significant, indicating a positive association between prior depressive episodes and negative explicit self-depressed associations. When neuroticism was added into the equation, the relationship between number of prior episodes and explicit self-associations was no longer significant. The final equation indicated that neuroticism, residual depressive symptoms, and implicit self-associations all showed independent predictive validity for the strength of explicit self-depressed associations (see Table 4). Also gender showed independent predictive value for the strength of explicit self-depressed associations. This

Table 3

Correlations Between Explicit and Implicit Measures at Baseline and 2-Year Follow-Up $(\mathrm{n}=726)$

\begin{tabular}{lccc}
\hline \multicolumn{1}{c}{ Measure } & 2 & 3 & 4 \\
\hline 1. EA T0 & $.56^{*}$ & $.31^{*}$ & $.19^{*}$ \\
2. EA follow-up & - & $.20^{*}$ & $.30^{*}$ \\
3. IAT T0 & - & - & $.52^{*}$ \\
4. IAT follow-up & - & - & - \\
\hline
\end{tabular}

Note. $\mathrm{IAT}=$ Implicit Association Test; $\mathrm{T} 0=$ baseline; $\mathrm{EA}=$ explicit associations.

${ }^{*}$ Correlation significant at the 0.01 level (two-tailed) might indicate that women are more likely to explicitly associate themselves with depression.

\section{Is the Duration of Depressive Symptoms Between Baseline and 2-Year Follow-Up Predictive for Implicit Self-Depressed Associations at Follow-Up?}

Multiple predictor regression analyses showed that greater amounts of time during which individuals suffered from depressive symptoms between baseline and follow-up was significantly associated with stronger implicit self-depressed associations at follow-up, even when we corrected for the depression IAT at baseline (see Table 5). Furthermore, this remained so, when we added neuroticism into the regression model. Yet, when the IDS-SR was included in the model to correct for depressive symptoms at follow-up, the relationship between duration and implicit self-associations was no longer significant. In addition, independent of IDS scores, level of neuroticism, and duration of symptoms, women tended to show stronger implicit self-depressed associations than men, as was evidenced by gender showing a borderline significant value for the strength of implicit selfdepressed associations.

\section{Is the Duration of Depressive Symptoms Between Baseline and 2-Year Follow-Up Predictive for Explicit Self-Depressed Associations at Follow-Up?}

Multiple regression analyses showed that longer duration of depressive symptoms between baseline (T0) and follow-up at 24 months (T1) was significantly associated with stronger explicit

\footnotetext{
${ }^{1}$ Note that the previous study by Glashouwer \& de Jong (2010) did not find a significant association between the number of prior episodes and the strength of self-depressed associations. However, the prior study selected a smaller subgroup $(n=330)$ and, because the beta values were very similar, the nonsignificance of the relationship between the number of prior episodes and the strength of self-depressed associations in this prior study was probably due to a lack of power.
} 
Table 4

Multiple Regression Analyses: Number of Prior Depressive Episodes Predicting the Strength of Implicit and Explicit Self-Depressed Associations at Baseline $(\mathrm{n}=666)$

\begin{tabular}{|c|c|c|c|c|c|c|c|}
\hline $\begin{array}{l}\text { Dependent variable IAT D-measure } \\
\text { Included }\end{array}$ & $\beta$ & $t$ & $p$ & $\begin{array}{c}\text { Dependent variable explicit measure } \\
\text { Included }\end{array}$ & $\beta$ & $t$ & $p$ \\
\hline Model 1* & & & & Model 1* & & & \\
\hline No. of episodes & -.09 & -2.54 & $<.01$ & No. of episodes & -.14 & -3.87 & $<.01$ \\
\hline Model 2* & & & & Model 2* & & & \\
\hline No. of episodes & -.07 & -1.88 & .06 & No. of episodes & -.04 & -1.42 & .15 \\
\hline Neuroticism & -.15 & -4.10 & $<.01$ & Neuroticism & -.66 & -22.33 & $<.01$ \\
\hline Model 3 & & & & Model 3* & & & \\
\hline No. of episodes & -.07 & -1.79 & .07 & No. of episodes & -.02 & -0.73 & .46 \\
\hline Neuroticism & -.13 & -0.25 & .01 & Neuroticism & -.46 & -12.25 & $<.01$ \\
\hline IDS-SR & -.04 & -0.75 & .45 & IDS-SR & -.28 & -7.57 & $<.01$ \\
\hline Model 4* & & & & Model 4* & & & \\
\hline No. of episodes & -.06 & -1.71 & .08 & No. of episodes & -.01 & -0.50 & .61 \\
\hline Neuroticism & -.05 & -0.86 & .38 & Neuroticism & -.45 & -11.96 & $<.01$ \\
\hline IDS-SR & .01 & 0.20 & .83 & IDS-SR & -.28 & -7.53 & $<.01$ \\
\hline EA T0 & .17 & 3.30 & .01 & IAT T0 & .09 & 3.30 & .01 \\
\hline Model 5 & & & & Model $5^{*}$ & & & \\
\hline No. of episodes & -.06 & -1.70 & .08 & No. of episodes & .00 & -0.10 & .91 \\
\hline Neuroticism & -.04 & -0.81 & .41 & Neuroticism & -.47 & -12.38 & $<.01$ \\
\hline IDS-SR & $<.01$ & 0.15 & .87 & IDS-SR & -.27 & -6.92 & $<.01$ \\
\hline EA T0 & .17 & 3.32 & $<.01$ & IAT T0 & .09 & 3.32 & $<.01$ \\
\hline Age & -.02 & -0.56 & .57 & Age & -.04 & -1.45 & .14 \\
\hline Gender & -.02 & -0.72 & .46 & Gender & .09 & 3.49 & $<.01$ \\
\hline Years of education & -.01 & -0.41 & .67 & Years of education & .00 & 0.12 & .89 \\
\hline$R^{2}=.01$ & .01 & 0.41 & .01 & $R^{2}=.02$ & & & \\
\hline$\Delta R^{2}=.02$ & & & & $\Delta R^{2}=.42$ & & & \\
\hline$\Delta R^{2}=.001$ & & & & $\Delta R^{2}=.04$ & & & \\
\hline$\Delta R^{2}=.01$ & & & & $\Delta R^{2}=.00$ & & & \\
\hline$\Delta R^{2}=.00$ & & & & $\Delta R^{2}=.01$ & & & \\
\hline
\end{tabular}

Note. $\quad$ IAT $=$ Implicit Association Test; Neuroticism $=$ Neuroticism domain of the NEO-Five Factor Inventory; IDS-SR $=$ Inventory of Depressive Symptoms-Self-Report; EA = explicit associations; T0 = baseline.

* Significance of $F$ change $<.05$.

self-depressed-associations at follow-up, even when corrected for the strength of the explicit self-associations at baseline (see Table 5). This remained so, even when we added neuroticism into the regression model. Also, when the IDS-SR was included in the model to correct for depressive symptoms at follow-up, the relationship between explicit self-associations and duration remained significant. When we controlled for the implicit measure, the relationship between duration of depressive symptoms and explicit self-depressed associations remained significant. Years of education showed a significant association with the strength of the explicit self-depressed associations. This unexpected finding seems to indicate that people with more education are more resistant to explicitly associate themselves with depression.

\section{Discussion}

The present study represents a critical first step in testing the hypothesis that more frequent depressive episodes or longer duration of depressive symptoms results in stronger implicit and/or explicit self-depressed associations ("scars"). The main results of this study can be summarized as follows: (a) the number of prior depressive episodes was positively related to the strength of both implicit and explicit self-depressed associations at baseline; (b) when controlling for neuroticism and depressive symptoms, for explicit self-depressed associations this relationship disappeared whereas for implicit self-associations the relationship just fell short of the conventional level of significance; (c) the duration of depressive symptoms between baseline and 2-year follow-up showed positive predictive value for the strength of explicit and implicit self-depressed associations at follow-up, over and above self-depressed associations during the baseline assessment; (d) even when neuroticism was taken into account, the duration of depressive symptoms still showed positive predictive value for the strength of the implicit as well as the explicit self-depressed associations at follow-up; and (e) when depressive symptoms were also taken into account, the duration of depressive symptoms was still a predictor of the strength of explicit-but no longer of implicit—self-depressed associations.

Previous research has provided tentative evidence for the view that negative self-associations become increasingly ingrained by repeated activation of negative self-associations during recurrent depressive episodes (Risch et al., 2010). In a series of exploratory analyses, Risch et al. found that remitted patients with three or more depressive episodes showed more negative self-associations than remitted patients with one or two former depressive episodes (although it should be acknowledged that the difference between never depressed controls and remitted patients was not significant, probably due to lack of sufficient statistical power). Building further on this theoretically important preliminary finding, the first aim of this study was to see whether we could replicate the earlier 
Table 5

Multiple Regression Analyses: Duration of Depressive Symptoms Between Baseline and 2-Year Follow-Up Predicting the Strength of the Implicit and Explicit Self-Depressed Associations at 2-Year Follow-Up $(\mathrm{n}=726)$

\begin{tabular}{|c|c|c|c|c|c|c|c|}
\hline $\begin{array}{c}\text { Dependent variable IAT D measure } \\
\text { Included }\end{array}$ & $\beta$ & $t$ & $p$ & $\begin{array}{l}\text { Dependent variable explicit measure } \\
\text { Included }\end{array}$ & $\beta$ & $t$ & $p$ \\
\hline Model $1^{*}$ & & & & Model $1^{*}$ & & & \\
\hline Duration* & -.14 & -3.88 & $<.01$ & Duration & -.36 & -10.55 & $<.01$ \\
\hline Model 2 & & & & Model 2* & & & \\
\hline Duration & -.08 & -2.74 & $<.01$ & Duration & -.24 & -7.87 & $<.01$ \\
\hline IAT T0 & .51 & 16.35 & $<.01$ & EA T0 & .50 & 16.46 & $<.01$ \\
\hline Model 3 & & & & Model 3* & & & \\
\hline Duration & -.08 & -2.62 & $<.01$ & Duration & -.23 & -7.85 & $<.01$ \\
\hline IAT T0 & .51 & 15.72 & $<.01$ & EA T0 & .44 & 11.79 & $<.01$ \\
\hline Neuroticism & -.02 & -6.07 & .54 & Neuroticism & -.09 & -2.50 & .01 \\
\hline Model 4* & & & & Model 4* & & & \\
\hline Duration & -0.02 & -0.82 & .41 & Duration & -.10 & -3.80 & $<.01$ \\
\hline IAT T0 & .50 & 15.87 & $<.01$ & EA T0 & .35 & 11.06 & $<.01$ \\
\hline Neuroticism & .03 & 0.93 & .34 & Neuroticism & .00 & -0.17 & .86 \\
\hline IDS-S* ${ }^{*}$ & -.18 & -5.2 & $<.01$ & IDS-SR & -.48 & -17.00 & $<.01$ \\
\hline Model 5 & & & & Model $5^{*}$ & & & \\
\hline Duration & -.004 & -0.10 & .91 & Duration & -.09 & -3.64 & $<.01$ \\
\hline IAT T0 & .49 & 15.58 & $<.01$ & EA T0 & .34 & 10.87 & $<.01$ \\
\hline Neuroticism & .06 & 1.85 & .06 & Neuroticism & .00 & -0.02 & .97 \\
\hline IDS-SR & -.09 & -2.21 & .02 & IDS-SR & -.46 & -16.25 & $<.01$ \\
\hline EA T1 & .17 & 4.08 & $<.01$ & IAT T1 & .11 & 4.45 & $<.01$ \\
\hline Model 6 & & & & Model $6^{*}$ & & & \\
\hline Duration & .00 & -.22 & .82 & Duration & -.09 & -3.53 & $<.01$ \\
\hline IAT T0 & .48 & 15.03 & $<.01$ & EA T0 & .34 & 10.75 & $<.01$ \\
\hline Neuroticism & .05 & 1.5 & .13 & Neuroticism & .00 & -0.14 & .88 \\
\hline IDS-SR & -.08 & -1.98 & .04 & IDS-SR & -.47 & -16.55 & $<.01$ \\
\hline EA T1 & .17 & 4.18 & $<.01$ & IAT T1 & .11 & 4.65 & $<.01$ \\
\hline Age & -.04 & -1.27 & .20 & Age & .03 & 1.54 & .12 \\
\hline Gender & -.06 & -1.93 & .05 & Gender & .04 & 1.65 & .09 \\
\hline Years of education & -.02 & -0.94 & .34 & Years of education & -.07 & -2.99 & $<.01$ \\
\hline$R^{2}=.02$ & & & & $R^{2}=.13$ & & & \\
\hline$\Delta R^{2}=.26$ & & & & $\Delta R^{2}=.23$ & & & \\
\hline$\Delta R^{2}=.00$ & & & & $\Delta R^{2}=.00$ & & & \\
\hline$\Delta R^{2}=.02$ & & & & $\Delta R^{2}=.17$ & & & \\
\hline$\Delta R^{2}=.01$ & & & & $\Delta R^{2}=.01$ & & & \\
\hline$\Delta R^{2}=.00$ & & & & $\Delta R^{2} \leq .01$ & & & \\
\hline
\end{tabular}

Note. $\quad$ IAT $=$ Implicit Association Test; T0 = baseline; EA = explicit associations; Neuroticism $=$ Neuroticism domain of the NEO-Five Factor Inventory; IDS-SR = Inventory of Depressive Symptoms-Self-Report; T1 $=24$ months.

${ }^{*}$ Significance of $F$ change $<.01$.

finding in an independent sample with sufficient statistical power to detect even relevant differences of small effect size. Corroborating these earlier findings, the retrospective single predictor regression analyses of the present study showed a significant relationship between the number of prior depressive episodes and the strength of the implicit and explicit self-depressed associations. These findings are consistent with the view that repeated depressive episodes may result in a scar, which in turn may lower the threshold for the recurrence of depressive episodes, eventually leading into a downward spiral (Glashouwer, de Jong, \& Penninx, 2012).

We reported that the relationships between the number of prior depressive episodes and self-depressed associations were no longer significant when we corrected for individual differences in neuroticism during baseline, although there was still a trend ( $p=$ .06) specifically for the implicit self-depressed associations. On the basis of this retrospective analysis alone, we cannot rule out that self-depressed associations are partly epiphenomenal to neuroticism. Another more general potential limitation of the retro- spective approach (which was also followed by Risch et al., 2010) is that the stronger self-depressed associations could reflect a premorbid characteristic instead of a scar. Therefore, we complemented the retrospective approach with a prospective approach that allowed us to control for the initial strength of the selfdepressed associations during the baseline assessment.

The results of this prospective approach were-like the retrospective findings - consistent with the view that depressive episodes may strengthen individuals' self-depressed associations. More specifically, the duration of depressive symptoms between baseline and 2-year follow-up showed positive predictive value for the strength of explicit and implicit self-depressed associations at follow-up, over and above self-depressed associations during the baseline assessment. Thus, these findings support the view that stronger self-depressed associations do not merely reflect premorbid characteristics, but rather that they are associated with previous depressive episodes. Of import, the duration of depressive symptoms still showed positive predictive value for the (implicit and explicit) self-depressed associations, even when neuroticism was 
taken into account. Thus, the results of the prospective analyses seem to suggest that self-depressed associations are not merely epiphenomenal to neuroticism.

Although the relationship between the duration of symptoms and self-depressed associations seemed largely independent of neuroticism, for the implicit self-associations, this relationship was no longer significant when correcting for depressive symptoms. This might imply that the relationship with the IAT was merely a byproduct of enhanced depressive symptomatology. However, given that enhanced implicit self-depressed associations may be a cause instead of a consequence of depressive symptoms, the inclusion of IDS in the regression equation might be considered as too stringent a test. Unfortunately, because of the correlational design of the present study, we cannot determine whether stronger negative associations are due to longer prior symptom duration or merely reflect higher levels of current symptoms. A possible strategy to control for current depressive symptoms in future research would be to neutralize depressive mood before the IAT assessment, using a positive mood induction.

Based on the earlier findings of Risch et al. (2010) and the alleged reciprocal relationship between implicit and explicit selfassociations, we anticipated that the relationship between the number of prior episodes and duration of symptoms would be largely similar for both types of self-associations. In support of their interrelationship, the implicit and explicit measures of selfdepressed associations correlated significantly, both at baseline and at follow-up, with a small to moderate effect size $(r=0.3)$. Moreover, for both the retrospective and prospective analyses, the single predictor models showed a very similar pattern for implicit and explicit self-associations. However, the final models (also including depressive symptoms and neuroticism) showed a differential pattern, suggesting that different processes might be involved in the generation of implicit and explicit self-depressed associations.

Most notably, the prospective analyses provided strong evidence that the relationship between the duration of symptoms and the explicit self-depressed associations was at least partly independent of depressive symptoms, neuroticism, and other potentially relevant background variables. By contrast, the duration of symptoms showed no independent predictive value for the implicit selfdepressed associations. Adding further to the relative independency of implicit and explicit self-associations, the results of the prospective analyses indicated that the relationship between the duration of symptoms and the explicit self-associations remained largely unaffected by the inclusion of implicit self-associations in the equation.

One explanation for the absence of an independent relationship between implicit self-associations and duration of symptoms could be that the time window of 2 years might have been too small for self-associations to become sufficiently strong to become evident in the IAT. In line with this, there was a (marginally significant) independent relationship between implicit self-associations and the number of prior depressive episodes in the retrospective analyses, which covered a much larger time window.

The finding in the prospective analysis that the duration of symptoms was related to the strength of explicitly reported selfdepressed associations, whereas for the retrospective analysis there was no relationship between the number of prior episodes and explicit self-depressed associations when we controlled for neu- roticism and depressive symptoms, might be explained by differences between the samples. Participants in the retrospective analyses were all in remission during the assessments of the implicit and explicit associations. The absence of a current depression may have reduced the explicit tendency to associate oneself with depression and enhanced participants' motivation to correct the more implicit self-depressed associations in the process of validation. By contrast, for those in the prospective analyses who were also in remission at the time of the assessment, the time that elapsed after recovery from a depressive episode was very limited (2 years at most). Thus, even for this subgroup, the tendency to connect themselves with depression might still be stronger than in the remitted participants of the retrospective analyses. It seems, therefore, more appropriate to interpret the enhanced explicit self-depressed associations as a residual symptom than as a remaining scar.

All in all, the present pattern of findings is consistent with the view that depressive episodes may give rise to persistent dysfunctional self-associations. For a more comprehensive test of Beevers's model (2005), an important next step would be to investigate the mediating role of self-depressed associations in the relationship between the number (or duration) of prior episodes and the recurrence of depression. On the basis of previous research (see Beshai, Dobson, Bockting, \& Quigley, 2011, for a review), the length of follow-up in the present study seemed sufficient to justify conducting these mediation analyses in the present sample. However, in contrast to these earlier findings, there was no evidence for a direct relationship between number of prior episodes and risk of recurrence in this 2-year time period within the present study. ${ }^{2}$ In the absence of such a relationship, it was not meaningful to test the mediating role of baseline self-depressed associations within the current sample. It would be important for future research to use a longer follow-up period, because it may take longer for the impact of prior episodes on the rate of recurrence to become evident.

The results of our study were strongest and most consistent for the explicit associations. Following these results, at least from a pragmatic perspective, it would seem most appropriate to focus on explicit self-depressed associations. An important next step would be to follow an experimental approach designed specifically to reduce explicit and/or implicit self-depressed associations (cf. Clerkin \& Teachman, 2010), and to test whether such manipulations would reduce the chance of recurrence in remitted patients. If explicit (and/or implicit) self-depressed associations are crucially involved in the recurrence of symptoms, experimentally reducing explicit (and/or implicit) self-depressed associations should result in a lower probability of recurrence of depressive symptoms. Recently, Clerkin and Teachman (2010) showed that a computerized association task was effective in reducing social anxiety by modifying participants' dysfunctional associations. Accordingly, it would be interesting to examine whether such a computer-based intervention might also be helpful to weaken self-depressed associations in remitted depressed patients and, thereby, to prevent relapse and recurrence in depression (cf. Vasey, Harbaugh, Buffington, Jones, \& Fazio, 2012). If so, this would not only provide more direct support for the (causal) role of self-depressed associ-

\footnotetext{
2 The analysis of the direct relationship between the number of prior depressive episodes and risk of recurrence in this 2-year time period $(n=$ $606)$ showed that the coefficient was $.00(S E=.01, p=.83)$.
} 
ations in the recurrent nature of depression, but it would also provide a fresh theory-derived clinical tool to break the highly invalidating rhythm of depression.

\section{References}

American Psychiatric Association. (2001). Diagnostic and statistical manual of mental disorders (4th ed., text revision). Washington, DC: American Psychiatric Association.

Back, M. D., Schmukle, S. C., \& Egloff, B. (2009). Predicting actual behavior from the explicit and implicit self-concept of personality. Journal of Personality and Social Psychology, 97, 533-548. doi: $10.1037 / \mathrm{a} 0016229$

Beevers, C. G. (2005). Cognitive vulnerability to depression: A dual process model. Clinical Psychology Review, 25, 975-1002.

Beshai, S., Dobson, K. S., Bockting, C. L. H., \& Quigley, L. (2011). Relapse and recurrence prevention in depression: Current research and future prospects. Clinical Psychology Review, 31, 1349-1360. doi: 10.1016/j.cpr.2011.09.003

Clark, D. A., Beck, A. T., \& Alford, B. A. (1999). Scientific foundations of cognitive theory and therapy of depression. New York, NY: Wiley.

Clerkin, E. M., \& Teachman, B. A. (2010). Training implicit social anxiety associations: An experimental intervention. Journal of Anxiety Disorders, 24, 300-308. doi:10.1016/j.janxdis.2010.01.001

Gawronski, B., \& Bodenhausen, G. V. (2006). Associative and propositional processes in evaluation: An integrative review of implicit and explicit attitude change. Psychological Bulletin, 132, 692-731.

Glashouwer, K. A., \& de Jong, P. J. (2010). Disorder-specific automatic self-associations in depression and anxiety: Results of the Netherlands Study of Depression and Anxiety. Psychological Medicine, 40, 11011111. doi:10.1017/S0033291709991371

Glashouwer, K. A., de Jong, P. J., \& Penninx, B. W. J. H. (2012). Prognostic value of implicit and explicit self-associations for the course of depressive and anxiety disorders. Behaviour Research and Therapy, 50, 479-486. doi:10.1016/j.brat.2012.05.002

Glashouwer, K. A., Smulders, F. T. Y., de Jong, P. J., Roefs, A., \& Wiers, R. W. (2013). Measuring automatic associations: Validation of algorithms for the Implicit Association Test (IAT) in a laboratory setting. Journal of Behavior Therapy and Experimental Psychiatry, 44, 105113. doi:10.1016/j.jbtep.2012.07.015

Greenwald, A. G., McGhee, D. E., \& Schwartz, J. L. K. (1998). Measuring individual differences in implicit cognition: The Implicit Association Test. Journal of Personality and Social Psychology, 74, 1464-1480.

Greenwald, A. G., Nosek, B. A., \& Banaji, M. R. (2003). Understanding and using the Implicit Association Test: I. An improved scoring algorithm. Journal of Personality and Social Psychology, 85, 197-216.

Haeffel, G. J., Abramson, L. Y., Brazy, P. C., Shah, J. Y., Teachman, B. A., \& Nosek, B. A. (2007). Explicit and implicit cognition: A preliminary test of a dual-process theory of cognitive vulnerability to depression. Behaviour Research and Therapy, 45, 1155-1167.

Judd, L. L., Akiskal, H. S., Zeller, P. J., Endicott, J., Coryell, W., Paulus, M. P., . . . Keller, M. B. (1998). A prospective 12-year study of subsyndromal and syndromal depressive symptoms in unipolar major depressive disorders. Archives of General Psychiatry, 55, 694-700.

Lamers, F., Hoogendoorn, A., Smit, J. H., van Dyck, R., Zitman, F. G., Nolen, W. A., \& Penninx, B. W. J. H. (2012). Sociodemographic and psychiatric determinants of attrition in the Netherlands Study of Depression and Anxiety (NESDA). Comprehensive Psychiatry, 53, 63-70. doi:10.1016/j.comppsych.2011.01.011

Lyketsos, C. G., Nestadt, G., Cwi, J., \& Heithoff, K. (1994). The Life Chart Interview: A standardized method to describe the course of psychopa- thology. International Journal of Methods in Psychiatric Research, 4 143-155.

Mathers, C. D., \& Loncar, D. (2006). Projections of global mortality and burden of disease from 2002 to 2030. PLoS Medicine, 3, e442.

McCrae, R. R., \& Costa, P. T., Jr. (2004). A contemplated revision of the NEO Five-Factor Inventory. Personality and Individual Differences, 36, 587-596.

Mueller, T. I., Leon, A. C., Keller, M. B., Solomon, D. A., Endicott, J., Coryell, W., . . . Maser, J. D. (1999). Recurrence after recovery from major depressive disorder during 15 years of observational follow-up. The American Journal of Psychiatry, 156, 1000-1006.

Ormel, J., Petukhova, M., Chatterji, S., Aquilar-Gaxioloa, S., Alonso, J., Angermeyer, M. C., . . . Kessler, R. C. (2008). Disability and treatment of specific mental and physical disorders across the world. The British Journal of Psychiatry, 192, 368-375. doi:10.1192/bjp.bp.107.039107

Penninx, B. W. J. H., Beekman, A. T. F., Johannes, H. S., Zitman, F. G., Nolen, W. A., Spinhoven, P., . . NESDA Research Consortium. (2008), The Netherlands Study of Depression and Anxiety (NESDA): Rationale, objectives and methods. International Journal of Methods in Psychiatric Research, 17, 121-140. doi:10.1002/mpr.256

Penninx, B. W. J. H., Nolen, W. A., Lamers, F., Zitman, F. G., Smit, J. H. Spinhoven, P., . . Beekman, A. T. (2011). Two-year course of depressive and anxiety disorders: Results from the Netherlands Study of Depression and Anxiety (NESDA). Journal of Affective Disorders, 133, 76-85. doi:10.1016/j.jad.2011.03.027

Risch, A. K., Buba, A., Birk, U., Morina, N., Steffens, M. C., \& Stangier, U. (2010). Implicit self-esteem in recurrently depressed patients. Journal of Behavior Therapy and Experimental Psychiatry, 41, 199-206. doi: 10.1016/j.jbtep.2010.01.003

Robins, L. N., Wing, J., Wittchen, H. U., Helzer, J. E., Babor, T. F., Burke, J., . . Towle, L. H. (1988). The Composite International Diagnostic Interview. An epidemiologic instrument suitable for use in conjunction with different diagnostic systems and in different cultures. Archives of General Psychiatry, 45, 1069-1077. doi:10.1001/archpsyc.1988 01800360017003

Rush, A. J., Gullion, C. M., Basco, M. R., Jarrett, R. B., \& Trivedi, M. H. (1996). The Inventory of Depressive Symptomatology (IDS): Psychometric properties. Psychological Medicine, 26, 477-486.

Smit, F., Cuijpers, P., Oostenbrink, J., Batelaan, N., de Graaf, R., \& Beekman, A. (2006). Costs of nine common mental disorders: Implications for curative and preventive psychiatry. The Journal of Mental Health Policy and Economics, 9, 193-200.

Spinhoven, P., Elzinga, B., Roelofs, K., Hovens, J. G. F. M., van Oppen, P., Zitman, F. G., \& Penninx, B. J. W. H. (2011a). The effects of neuroticism, extraversion, and positive and negative life events on a one-year course of depressive symptoms in euthymic previously depressed patients versus healthy controls. The Journal of Nervous and Mental Disease, 199, 684-689. doi:10.1097/NMD.0b013e318229d21f

Spinhoven, P., Elzinga, B., Roelofs, K., Hovens, J. G. F. M., van Oppen, P., Zitman, F. G., \& Penninx, B. J. W. H. (2011b). Personality, life events and the course of anxiety and depression. European Journal of Personality, 124, 443-452. doi:10.1111/j.1600-0447.2011.01753.x

Vasey, M. W., Harbaugh, C. N., Buffington, A. G., Jones, C. R., \& Fazio, R. H. (2012). Predicting return of fear following exposure therapy with an implicit measure of attitudes. Behaviour Research and Therapy, 50, 767-774. doi:10.1016/j.brat.2012.08.007

Wacker, H. R., Battegay, R., Muellejans, R., \& Schlosser, C. (2006). Using the CIDI-C in the general population. In C. N. Stefanis, A. D. Rabavilas, \& C. R. Soldatos (Eds.), Psychiatry: A world perspective (pp. 138-143). Amsterdam, The Netherlands: Elsevier Science. 


\section{Appendix A}

\section{Implicit Association Test Stimulus Words}

Me: I, myself, self, my, own

Other: other, you, they, them, themselves

Depressed: useless, pessimistic, inadequate, negative, meaningless

Elated: positive, optimistic, active, valuable, cheerful

Words are translated from Dutch

Received October 3, 2012

Revision received September 30, 2013

Accepted October 4, 2013

\section{Call for Nominations}

The Publications and Communications (P\&C) Board of the American Psychological Association has opened nominations for the editorships of History of Psychology; Journal of Family Psychology; Journal of Personality and Social Psychology: Personality Processes and Individual Differences; Psychological Assessment; Psychological Review; International Journal of Stress Management; and Personality Disorders: Theory, Research, and Treatment for the years 2016-2021. Wade Pickren, PhD, Nadine Kaslow, PhD, Laura King, PhD, Cecil Reynolds, PhD, John Anderson, PhD, Sharon Glazer, $\mathrm{PhD}$, and Carl Lejuez, PhD, respectively, are the incumbent editors.

Candidates should be members of APA and should be available to start receiving manuscripts in early 2015 to prepare for issues published in 2016. Please note that the P\&C Board encourages participation by members of underrepresented groups in the publication process and would particularly welcome such nominees. Self-nominations are also encouraged.

Search chairs have been appointed as follows:

- History of Psychology, David Dunning, $\mathrm{PhD}$

- Journal of Family Psychology, Patricia Bauer, PhD, and Suzanne Corkin, PhD

- JPSP: Personality Processes and Individual Differences, Jennifer Crocker, PhD

- Psychological Assessment, Norman Abeles, PhD

- Psychological Review, Neal Schmitt, PhD

- International Journal of Stress Management, Neal Schmitt, PhD

- Personality Disorders: Theory, Research, and Treatment, Kate Hays, $\mathrm{PhD}$, and Jennifer Crocker, PhD

Candidates should be nominated by accessing APA's EditorQuest site on the Web. Using your Web browser, go to http://editorquest.apa.org. On the Home menu on the left, find "Guests." Next, click on the link "Submit a Nomination," enter your nominee's information, and click "Submit."

Prepared statements of one page or less in support of a nominee can also be submitted by e-mail to Sarah Wiederkehr, P\&C Board Search Liaison, at swiederkehr@apa.org.

Deadline for accepting nominations is January 11, 2014, when reviews will begin. 